\title{
Frustrated Magnetism on Nd-based Shastry-Sutherland (SS) lattices
}

Gabriele Sala (gabrysala86@hotmail.it)

Matthew Stone (stonemb@ornl.gov)

Andrew Christianson (christiansad@ornl.gov)

The interactions that define how spins arrange themselves in a material play a fundamental role in a wide variety of physical phenomena. The combinations of single ion anisotropies, exchange interactions and lattice defects offer to physicists a proving ground for testing old and new theories. This is especially relevant in frustrated magnetism, where there are still open questions and challenges to resolve. Geometrically frustrated magnets are systems in which the competing interactions governing the spins cannot be simultaneously satisfied, leading to a highly degenerate ground state and the manifestation of exotic properties. Historically, the triangular lattice with antiferromagnetic interactions has been the prototype to study this phenomenon and, over the past decades, many 2D and 3D systems were discovered to posses frustration as a key ingredient to study their properties. In this presentation, I will focus on the Shastry-Sutherland lattice, a model 2D frustrated system for quantum magnetism which is exactly solvable. By means of neutron scattering techniques, bulk measurements and crystal field analysis, I will compare two distinct examples of Nd-based compounds that host novel physics: the metallic Ising anti-ferromagnet $\mathrm{Nd}_{2} \mathrm{Ni}_{2} \mathrm{In}$, and $\mathrm{BaNd}_{2} \mathrm{ZnO}_{5}$ which hosts ferromagnetic dimers. Although the underling lattice of moments is similar in these two compounds, the details of the local environment of each compound manifests different behaviour in regards to magnetic properties. 